\title{
Fazit und Diskussion
}

Die vorliegende Arbeit hat sich mit den Fragen befasst, über welche habituellen Dispositionen die Lehrkräfte im Hinblick auf die Unterstützung sozial benachteiligter und bisher im Bildungssystem ausgegrenzter Schüler*innen verfügen und inwiefern diese handlungsleitenden Orientierungen milieuspezifisch eingebettet sind. Ziel der Arbeit war es, Passungskonstellationen zwischen Lehrkräften und Schüler*innen vertiefend zu analysieren und dabei nicht nur Formen der Differenzherstellung und Benachteiligung, sondern vor allem kompensatorische Bearbeitungsformen von Bildungsungleichheit in den Blick zu nehmen. Im Forschungsfokus standen somit bislang kaum untersuchte soziale Determinanten des Lehrer*innenhandelns im Kontext bereits vorhandener Ressourcen der pädagogischen Arbeit. Damit hat die Arbeit einen Beitrag zur Erforschung sozialer Ungleichheit im Bildungssystem sowie zur Identifikation von Möglichkeiten zur Verringerung von Bildungsnachteilen leisten wollen, die unmittelbar in den Wirkungsbereich der Lehrkräfte fallen.

In diesem Kapitel werden zunächst die zentralen Ergebnisse der empirischen Analysen zusammengefasst (Abschnitt 5.1). Anschließend werden auf Grundlage aller bisherigen Betrachtungen praktische Konsequenzen für die Bearbeitung von Bildungsungleichheit abgeleitet (Abschnitt 5.2). Letztendlich werden Grenzen der vorliegenden Untersuchung sowie Perspektiven für weitere Forschungsarbeiten aufgezeigt (Abschnitt 5.3).

\subsection{Zusammenfassung der zentralen Ergebnisse}

Durch die unternommenen Rekonstruktionen konnte die Vielfalt und Komplexität des pädagogischen Handelns im Themenfeld Bildungsungleichheit aufgezeigt 
werden. Die Untersuchungsergebnisse belegen, dass die Lehrkräfte in unterschiedlicher Weise für die Lernvoraussetzungen und Bedürfnisse der Schüler*innen sensibel sind und sich in einem Spannungsfeld zwischen Reproduktion und Abbau von sozialer Ungleichheit bewegen. Einerseits sind die Befunde der empirischen Analysen anschlussfähig an das eingangs vorgestellte Forschungsfeld zur Verstrickung der Lehrkräfte in Reproduktionsprozesse sozialer Ungleichheit. So konnten in allen vier Fallanalysen negative Stereotype und Defizitzuschreibungen an die Schüler*innen und Eltern resp. eine problemorientierte Sichtweise auf den familialen Hintergrund sozial benachteiligter Schüler*innen herausgearbeitet werden. Frau Akay und Frau Kamper problematisieren den großen Unterstützungsbedarf im Unterricht und in lebenspraktischen Angelegenheiten, das Fehlen von Regeln und Strukturen sowie mangelnde Förderung im Elternhaus. Die geringen sozioökonomischen Ressourcen stellen aus diesem Blickwinkel ein Hindernis für gelingende Sozialisations- und (Schul-)Bildungsprozesse dar. Im Einklang mit den Erkenntnissen von Fölker und Hertel (2015), Jäger (2014) sowie Wiezorek und Pardo-Puhlmann (2013) wurde in den Darstellungen deutlich, dass beide Lehrerinnen den Eltern grundlegende Erziehungskompetenzen absprechen bzw. diese zumindest infrage stellen. Des Weiteren zeichnet sich in den Fallanalysen von Frau Akay und Frau Kamper bereits ab, dass mit der negativ verallgemeinernden Perspektive hinsichtlich des familialen Hintergrundes und somit auch der sozialen Herkunft geringe Erwartungen an die Lernfähigkeit der Schüler*innen einhergehen. Zwar kann durch diese Arbeit die tatsächliche Wirkung der pädagogischen Deutungsmuster etwa mit Blick auf die Schulempfehlungen nicht geklärt werden, allerdings legt die Forschung zu Lehrer*innenurteilen, Underachievement und Cooling-Out-Prozessen nahe, dass durch die Defizitzuschreibungen primäre Herkunftseffekte verstärkt werden (vgl. Bos \& Pietsch, 2007; Nölle, Hörstermann, Krolak-Schwerdt, \& Gräsel, 2009; Uhlig, Solga, \& Schupp, 2009; Anders, McElvany, \& Baumert, 2010; Sorhagen, 2013).

Andererseits konnten durch die Orientierung an bereits vorhandenen Ressourcen der pädagogischen Arbeit pädagogische Sicht- und Herangehensweisen der Unterstützung sozial benachteiligter und bisher im Bildungssystem ausgegrenzter Schüler*innengruppen rekonstruiert werden. Fallweise distanzieren sich die Lehrkräfte von einer eingeschränkten Wahrnehmung von herkunftsspezifischen Differenzen und von als unveränderlich und entschieden gedeuteter ,Problemfälle'. Etwa stellt Frau Kamper anhand einer vermeintlich ungepflegten Schülerin die perspektivgebundene Konstruktion des Begriffs Problemschüler*in heraus. Sie toleriert aufgrund eigener Erfahrung das äußere Erscheinungsbild der Schülerin und wertet die „ungekämmt[en]“ und ,zerwühlten Haare“ (Z. 334 f.) nicht als problematisch, wohingegen ihre Kollegin die Schülerin als ,wirklich verwahrlost“ 
(Z. 339) wahrnimmt. Herr Jansen bezieht ebenfalls kritisch Stellung zu der Kategorie ,Problemschüler*in “ und verweist insbesondere darauf, dass Schüler*innen mit ADHS nicht zwingend dazuzuzählen sind. Mit ,täglich anecken ' und aggressivem Verhalten nennt Herr Jansen zusätzliche notwendige Bedingungen für den Status Problemschüler*innen und markiert zugleich, dass diese Kriterien temporär, graduell oder auch gar nicht zutreffen können bzw. dass dies keine dauerhafte Eigenschaft darstellt, sondern im Laufe der Zeit veränderbar ist.

Ferner wurde nachgewiesen, dass die Lehrkräfte auf vielfältige Weise bemüht sind, der sozialen Benachteiligungslage entgegenzuwirken und den Bedürfnissen der Schüler*innen nachzukommen. So kann die von Frau Akay und Frau Kamper vorgenommene Klassifikation und Kategorisierung von bedürftigen Kindern auch in einen anderen Bezugsrahmen gesetzt werden. Dadurch ist die defizit- und problemorientierte Perspektive der Lehrkräfte bezüglich der Erziehungsfähigkeit der Eltern nicht weniger kritisch zu betrachten, jedoch kann davon ausgegangen werden, dass jede Sichtweise oder Handlung in einem bestimmten Rahmen bzw. Kontext sinnstiftend ist. Etwa bietet Frau Akay den Kindern aus ihrer Perspektive „Sicherheit und Orientierung“ (Z. 481 f.) durch Rituale, Regeln und Tagesstrukturen in der Schule. Von beständigen und verlässlichen Strukturen in der Schule profitieren in der Regel jedoch nicht nur vermeintlich bedürftige, sondern alle Schüler*innen. Auch unterscheidet sie in dieser Weise bedürftige Kinder nicht von anderen. Frau Kamper orientiert ihr pädagogisches Handeln vor allem an dem Ausgleich wahrgenommener materieller Bedürftigkeit und kauft bzw. organisiert selbst Schulmaterialien für bedürftige Kinder. Durch diese Handlung werden zwar Benachteiligungsstrukturen nicht grundsätzlich verändert, jedoch erscheint das Vorgehen kurzfristig lösungsorientiert und ermöglicht eine planmäßige Fortsetzung des Unterrichts. Überdies ist Frau Kamper darauf bedacht, dass Kinder aufgrund des äußerlichen Auftretens nicht negativ im Klassenverband auffallen und berät demzufolge Eltern in alltagspraktischen Angelegenheiten wie Körperpflege und Kleidung. Zudem versuchen die Lehrkräfte eine durch Empathie, Nähe und Vertrauen gekennzeichnete Beziehung zu sozial benachteiligten Schüler*innen aufzubauen. Nach Ansicht von Frau Akay und Frau Antonova gelingt dies bei Schüler*innen mit Migrationshintergrund aufgrund des scheinbar gemeinsamen Erfahrungshintergrundes von allein. Eine ähnliche Orientierung weist Frau Kamper im Hinblick auf Schüler*innen in scheinbar schwierigen Lebenslagen auf. Infolge einer konstatierten gemeinsamen Problembetroffenheit und angenommener ähnlicher biografischer Erfahrungen gelingt es ihr zufolge, eine vertrauensvolle Beziehung zu diesen Schüler*innen aufbauen und die speziellen Problemlagen sensibler zu deuten und zu bearbeiten. Herr Jansen sorgt aus seiner Sicht generell für ein Arbeits- und Sozialklima, im Rahmen dessen die 
Schüler*innen möglichst effizient lernen können. Die empathische Umgangsweise und die Wertschätzung jenseits von Schulleistungen ist hierbei eine zentrale Ressource, um primäre Herkunftseffekte zu verringern (vgl. Gronostaj, Kretschmann, Westphal, \& Vock, 2015; Calmbach, Thomas, Borchard, \& Flaig, 2012; Hattie, 2008; Drucks, Osipov, \& Quenzel, 2010).

Als bedeutsamste Einflussfaktoren auf die Wahrnehmung der Schüler*innenund Elternschaft, auf das berufliche Selbstverständnis sowie auf die Gestaltung der Unterrichts- und Interaktionsprozesse haben sich die wirtschaftlichen, sozialen und kulturellen Rahmenbedingungen im Einzugsbereich der Schule sowie die biografischen Erfahrungen der Lehrkräfte erwiesen. In Anknüpfung an die Befunde von Fölker, Hertel und Pfaff (2015a; 2015b) sowie Jäger (2014) konnte die vorliegende Untersuchung zunächst verdeutlichen, dass der sozialräumliche Kontext der Schule zentral für die Konstruktion der Schüler*innen- und Elternschaft und somit auch für das an den Klientelbildern orientierte pädagogische Handeln ist. Frau Akay und Frau Kamper, die beide an Schulen mit einer ethnisch und sozial segregierten Schüler*innenschaft arbeiten, entwerfen insgesamt eine auf mehreren Ebenen außergewöhnlich hilfsbedürftige Klientel. Aus Sicht der beiden Lehrerinnen benötigen die Schüler*innen sowohl im Unterricht als auch in lebenspraktischen Angelegenheiten auffallend viel pädagogische Unterstützung. So stehen bei Frau Akay und Frau Kamper Praktiken des Ausgleichs der angenommenen sozioökonomischen Benachteiligungslage im Mittelpunkt der pädagogischen Arbeit. Im Gegensatz dazu konstruiert Frau Antonova, die an einer Schule mit einer überwiegend sozial privilegierten Schüler*innenschaft unterrichtet, eine besonders schulleistungsstarke und schulbildungseifrige Klientel. Angesichts der enormen Lernfähigkeit und der intrinsischen Motivation gestaltet Frau Anotonova ihren Unterricht abwechslungsreich und gibt kontinuierlich neue Lernanstöße, sodass die Schüler*innen ihr Lernpotenzial optimal entfalten können. Herr Jansen, der an einer Schule mit einer soziokulturell heterogenen Schüler*innenschaft tätig ist, thematisiert kaum die schulischen Leistungen bzw. die Schüler*innenschaft als Ganzes, sondern behandelt vor allem die Frage, inwiefern Herausforderungen, die mit Verhaltensauffälligkeiten in Verbindung stehen, in der pädagogischen Arbeit zu bewältigen sind. So richtet sich seine pädagogische Arbeit maßgeblich an dem (Selbst-)Anspruch von weitestgehender Unterstützung aller Schüler*innen aus. Er steht nicht nur den leistungsschwächeren Schüler*innen und Schüler*innen mit sonderpädagogischem Förderbedarf bzw. Verhaltensauffälligkeiten bei, sondern fördert darüber hinaus gezielt leistungsstärkere Schüler*innen.

Des Weiteren demonstrierten die Untersuchungsergebnisse ähnlich wie die Analysen des Arbeitszusammenhanges von Bremer, Lange-Vester und TeiwesKügler (vgl. Lange-Vester, 2015; Bremer \& Lange-Vester, 2014; Lange-Vester 
\& Teiwes-Kügler, 2014; Lange-Vester \& Teiwes-Kügler, 2013), dass die handlungsleitenden Orientierungen der Lehrkräfte stark mit milieuspezifischen Habitusformen verwoben sind und damit auch über den Einzelfall hinaus relevant sind. Insgesamt zeigen die hier vorgestellten Fälle ein breites Milieuspektrum, wobei sich die Lehrkräfte vor allem auf einer horizontalen Ebene nach dem Grad ihrer Selbstbestimmtheit bzw. ihrer Hierarchiegebundenheit unterscheiden. Verkürzt kann zusammengefasst werden, dass Frau Akay, die dem Milieu der eher modernen bildungsorientierten Aufsteiger*innen (Geiling, Gardemin, Meise, \& König, 2011) zugeordnet werden kann, hauptsächlich über Schüler*innen mit Migrationshintergrund spricht. Aufgrund ihres eigenen türkischen Migrationshintergrundes und Aufwachsens im sozialen Brennpunkt hat sich Frau Akay nicht im Zuge ihrer Bewerbung als Förderschullehrerin von einer Grundschule mit einem sehr hohen Anteil an Schüler*innen mit Migrationshintergrund sowie sozioökonomisch benachteiligten Schüler*innen abschrecken lassen. Im Gegenteil: Infolge ihrer biografischen Erfahrungen schreibt sie sich pragmatisches Handlungswissen und besondere Handlungskompetenzen im Umgang mit Schüler*innen mit Migrationshintergrund zu. Der pädagogischen Arbeit liegt eine große soziale Sensibilität zugrunde, die typisch für dieses Milieu ist (ebd.). Etwa nutzt Frau Akay ihren Erfahrungshintergrund, um den Schüler*innen mit Migrationshintergrund ein Vorbild zu sein. Frau Antonova, die dem eher konservativen Milieu der erfolgsorientierten Aufsteiger*innen (ebd.) angehört, befasst sich ebenfalls überwiegend mit dem Thema Migration. Die für dieses Milieu charakteristische leistungsindividualistische Grundhaltung (ebd.) durchzieht ihr Integrationsverständnis. Dabei stellt die Anpassungs- und Leistungsbereitschaft der eigenen Mutter im Zuge der Migration den Maßstab für alle Einwander*innen dar. Auch das pädagogische Handeln ist maßgeblich am Leistungsprinzip orientiert, so gestaltet Frau Antonova ihren Unterricht abwechslungsreich und kognitiv anregend. Hinzu kommt, dass sie aufgrund ihrer eigenen Einwanderungsgeschichte von spezifischem Wissen und besonderen Kompetenzen für den Umgang mit Schüler*innen mit Migrationshintergrund ausgeht. Frau Kamper, die aus dem Milieu der traditionellen kleinbürgerlichen Arbeitnehmer*innen (Vester, von Oertzen, Geiling, Hermann, \& Müller, 2001) stammt, thematisiert vor allem die pädagogische Arbeit mit sozioökonomisch benachteiligten Schüler*innen. Anlässlich ihrer lebensgeschichtlichen Erfahrungen von bescheidenen, restriktiven Lebensumständen und emotionaler Vernachlässigung durch die Eltern hat sie sich gezielt für ein Grundschullehramtsstudium und eine Schule, an der sozial schlechtergestellte Schüler*innen überrepräsentiert sind, entschieden. Die starke Orientierung an Sicherheit und festen Ordnungsstrukturen, die bedeutsam für dieses Milieu sind (ebd.), findet ihren Niederschlag in der pädagogischen Arbeit. 
Etwa wünscht sie sich verbindliche Regelungen hinsichtlich des Umgangs mit Schüler*innen aus prekären Lebensverhältnissen und problembelasteten Familien. Herr Jansen setzt sich vorrangig mit dem Themenfeld Integration von verhaltensauffälligen Schüler*innen auseinander. Sein eigenverantwortliches und prinzipiell solidarisches pädagogisches Handeln sowie die aktive Entwicklung von Professionalität sind kennzeichnend für das Milieu der modernen Arbeitnehmer*innen (ebd.). Den eigenen Grundsatz, Partizipation zu maximieren und Ausgrenzung zu minimieren, vertritt er dabei selbstsicher gegenüber Eltern, Kolleg*innen und Schulleitung.

Im Gegensatz zu den vorgestellten Untersuchungen von Bremer, Lange-Vester und Teiwes-Kügler verweisen die Habitus- und Milieuanalysen dieser Studie darüber hinaus in groben Zügen auf zwei Grundmuster der lehrkraftseitigen Unterstützung sozial benachteiligter Schüler*innen: ein anerzogenes, prinzipiengeleitetes Engagement wie im Fall von Herrn Jansen sowie ein aus scheinbar homologen biografischen Erfahrungen resultierendes Engagement wie in den Fällen von Frau Akay, Frau Antonova und Frau Kamper. Der Konstruktion eines konjunktiven Erfahrungsraumes kommt demnach eine hohe Bedeutung hinsichtlich des Verständnisses für die Bedürfnisse bestimmter Schüler*innengruppen, der Zuschreibung von besonderem Wissen und spezifischen Kompetenzen, der Erfahrung von Selbstwirksamkeit sowie der Bereitschaft, Verantwortung für die Entwicklung von Bildungschancen zu übernehmen, zu. Die Arbeit legt die Komplexität der handlungsleitenden Orientierungen der Lehrkräfte offen und berücksichtigt mit der Konstruktion eines konjunktiven Erfahrungsraumes eine Dimension, die bislang in diesem Kontext nicht beachtet wurde. Mit der Vorstellung konjunktiver Erfahrungen gehen jedoch auch Gefahren und Fallstricke für das pädagogische Handeln einher. Zum einen kann die unbedachte Übertragung des eigenen Erfahrungshintergrundes auf die Schüler*innen der Aufgeschlossenheit für fallspezifische Besonderheiten im Wege stehen. So bleibt in Bezug auf das neu eingewanderte polnische Mädchen die angenommene Gemeinsamkeit auf die Fremdheitserfahrung im Zuge der Migration beschränkt. Ungleiche familiale Ressourcen und Sozialisationsbedingungen sowie verschiedene Ausgangslagen in divergierenden Heimatländern bezieht Frau Antonova in ihre Überlegungen nicht ein. Zum anderen ist in allen drei Fällen eine Affinität zu den Schüler*innen zu erkennen, mit denen die Lehrerinnen sich über ihre lebensgeschichtlichen Erfahrungen verbunden fühlen. Diese Befunde kommen den Studien zu beruflichen Selbst- und Fremdkonzepten von Lehrkräften mit Migrationshintergrund gleich, die der Frage nachgehen, wie sich Lehrkräfte mit Migrationshintergrund hinsichtlich der bildungspolitischen Zuschreibung besonderer Kompetenzen für 
den Umgang mit Schüler*innen mit Migrationshintergrund positionieren und welches berufliches Selbstverständnis sie vor diesem Hintergrund entwerfen (vgl. Fabel-Lamla \& Klomfaß, 2014; Rotter, 2014; Georgi, Ackermann, \& Karakas, 2011). So resümieren Fabel-Lamla und Klomfaß (2014), dass eine habituelle Übereinstimmung zwischen Lehrkräften und Schüler*innen mit Migrationshintergrund in einzelnen Fällen zwar zu einer besonders vertrauensvollen Beziehung führen kann, dies aber kein angemessenes Fallverstehen und eine Zunahme des Bildungserfolgs von Schüler*innen mit Migrationshintergrund garantiert.

Insgesamt zeigen die Rekonstruktionen der vorliegenden Studie auf, dass die habitus- und milieuspezifischen Orientierungen der Lehrkräfte bezüglich der Unterstützung sozial benachteiligter und bisher im Bildungssystem ausgegrenzter Schüler differenzierter als vermutet erscheinen und mit der Konstruktion eines konjunktiven Erfahrungsraumes Facetten umfassen, die bislang in diesem Kontext nicht beachtet wurden. Zudem werden mit dieser Spielart des HomogamieEffektes in pädagogischen Beziehungen (u. a. Bourdieu \& Wacquant, 2006) der quantitativ ausgerichteten Forschung zum Rational-Choice-Ansatz starke Befunde entgegengesetzt. So kann mit der vorliegenden Arbeit empirisch belegt werden, dass der Blick auf und der Umgang mit Schüler*innen durchaus in einem engen Zusammenhang mit der sozialen Herkunft der Lehrkräfte steht und die pädagogische Arbeit vor allem für die Schüler*innenklientel förderlich zu sein scheint, die mehr Übereinstimmung mit den jeweiligen biografischen Erfahrungen und Haltungen der Lehrkräfte aufweisen.

\subsection{Handlungsoptionen für die Bearbeitung von Bildungsungleichheit}

Auf Grundlage des erkenntnistheoretischen Prinzips der Sozioanalyse von Pierre Bourdieu können anhand der Untersuchungsergebnisse Anhaltspunkte und Entwicklungsmöglichkeiten für eine rationale und (selbst-)reflexive pädagogische Arbeit hergeleitet werden. Generell geht es bei Bourdieus Konzept der Sozioanalyse darum, sich mit der eigenen sozialen Herkunft und somit mit den GesetzmäBigkeiten und (verinnerlichten) Restriktionen der eigenen Wahrnehmungs-, Denkund Handlungsmuster zu befassen (u. a. Bourdieu \& Wacquant, 2006; Bourdieu, 2002; 1993). Im Kontext der pädagogischen Arbeit bedeutet Sozioanalyse, soziale Ungleichheit zum Ausgangpunkt für weitere Überlegungen zur individuellen Förderung benachteiligter Schüler*innen und zur Verringerung ungleicher Bildungschancen zu nehmen. Einfache Antworten auf die Frage, wie eine sozioanalytische pädagogische Arbeit konkret auszusehen hat, gibt es nicht - dafür 
ist Bourdieus Programm zu anspruchsvoll und komplex. Die folgenden Ausführungen sind demnach nicht als konkrete Handlungsempfehlungen zu verstehen, sondern stellen vielmehr einen Reflexionsrahmen und Anregungen für weitere Auseinandersetzungen dar.

Zunächst zeigen die Einzelfallanalysen, dass das Reflexions- und Wissensniveau von Lehrkräften über Reproduktionsmechanismen sozialer Ungleichheit im Bildungssystem und die eigene Verstrickung in solche Prozesse insgesamt angehoben werden sollte (vgl. Lange-Vester \& Teiwes-Kügler, 2014). Übereinstimmend mit den Hinweisen von Rieger-Ladich (2011) und Ditton (2010a) kann in dieser Arbeit kein Bewusstsein der Lehrkräfte für ihre eigene Mitwirkung bei der Perpetuierung sozialer Ungleichheit rekonstruiert werden. Zwar nehmen alle vier Lehrkräfte grundsätzlich wahr, dass Chancengleichheit im Bildungssystem nur eingeschränkt gegeben ist, die Ursachen für die Bildungsnachteile einiger Schüler*innen verorten die befragten Lehrkräfte allerdings primär in der Familie. So spielen aus ihrer Sicht die Eltern eine tragende Rolle für den erfolgreichen Verlauf der Bildungsbiografie der Schüler*innen. Fallweise schätzen die Lehrkräfte auch institutionelle Rahmenbedingungen wie etwa die derzeitigen inadäquaten Voraussetzungen an allgemeinbildenden Schulen für gemeinsames Lernen (Herr Jansen) oder unzureichende Personalressourcen (Frau Kamper) als ursächlich für ungleiche Bildungschancen bzw. als Einflussgröße für Bildungsungleichheit ein. Die Betonung von externen kontextuellen Ursachen dient hierbei als Erklärung für Grenzen des eigenen pädagogischen Handelns. Die Fähigkeit und Bereitschaft der Lehrkräfte, die eigene pädagogische Praxis auch als symbolische Gewalt zu begreifen (vgl. Lange-Vester \& Teiwes-Kügler, 2014) scheint eine grundlegende Voraussetzung dafür zu sein, die Verantwortung für die Entwicklung von Bildungschancen zu stärken.

Weiterhin gilt es seitens der Lehrkräfte, differente Lebenswelten, Alltagskulturen, Werte und Einstellungen und daraus resultierende ungleiche Lernvoraussetzungen, erfahrungsweltliche Bildungsinhalte und -strategien der Schüler*innen wahrzunehmen und anzuerkennen. Dahingehend plädieren Grundmann, GrohSamberg, Bittlingmayer und Bauer (2003) für einen erweiterten Bildungsbegriff, der außerschulische Bildungsprozesse stärker fokussiert. Die Autoren argumentieren, dass das Wissen, die Fähigkeiten und Kompetenzen von Schüler*innen aus sozial benachteiligten Milieus nicht immer anschlussfähig an schulische, standardisierte Bildung sind und zum Teil außerwertgesetzt werden (ebd.). In die Diskussion um den Zusammenhang von Milieuzugehörigkeit und spezifischen Lern- und Bildungsformen ist auch die Forderung von Bremer (2007) nach einer „Enthierarchisierung von Kompetenzen“ (ebd., 268 ff.) zugunsten einer 
Stärkung und Wertschätzung von Interessen, Kenntnissen und Potenzialen speziell von Schüler*innen aus unterprivilegierten und aufstiegsorientierten Milieus einzuordnen. Etwa weisen Studien zu Bildungsaufstiegsprozessen auf die enormen Anpassungsleistungen der Aufsteiger*innen hin, die durch Lehrkräfte nicht unterstützt werden. Der soziale Aufstieg erfordert nicht nur in hohem Maße (Selbst-)Reflexion und Flexibilität, sondern auch eine ausgeprägte Trennungskompetenz. Zudem kann festgestellt werden, dass Aufsteiger*innen zahlreiche Hürden überwinden müssen: Die meisten können auf keine schulbildungsrelevante Hilfestellung zurückgreifen und müssen mitunter gegen Widerstand in der Familie bzw. im Herkunftsmilieu kämpfen (u. a. El-Mafaalani, 2012; von Rosenberg, 2011; King, 2009).

Auch wenn aus den unternommenen Rekonstruktionen hervorgeht, dass die Lehrkräfte nicht ausschließlich auf schulische Leistungen, sondern auf die Schüler*innen als ganze Person blicken und sensibel für ungleiche Ressourcen- bzw. Kapitalausstattungen sind, handelt es sich nicht um ein ,generelles und genetisches Verständnis der Existenzbedingungen“ (Bourdieu, 1987, S. 786). Im Allgemeinen basieren die dargelegten Kenntnisse über die soziale Situation der Schüler*innen und deren Auswirkungen auf den Schulkontext entweder auf eigenem Erfahrungswissen oder auf institutionalisierten Deutungsmustern. Bei ,Verstehen' im Sinne von Bourdieu geht es aber gerade nicht darum, sich aufgrund eigener biografischer Erfahrungen in bestimmte Schüler*innen emotional hineinversetzen und sich mit ihnen identifizieren zu können, sondern um die Einsicht, ,in die untrennbar verwobenen psychischen und sozialen Prägungen, die mit der Position und dem biographischen Werdegang dieser Person im Sozialraum einhergehen" (ebd.). Im Hinblick auf die negativen Schulerfahrungen von Frau Akay (Hauptschulzuweisung und institutionellen ,Haltekräfte‘ der Hauptschule), die als Musterbeispiel für die institutionelle Diskriminierung einer Schülerin mit Migrationshintergrund gedeutet werden können (vgl. Gomolla \& Radtke, 2009), soll der Unterschied herausgestellt werden: So ist zu vermuten, dass Frau Akay Erfahrungen von Differenz und Ungleichheit gemacht hat bzw. macht. Die eigenen Erlebnisse und die eigene Problembetroffenheit entlasten aber sobald es um die pädagogische Arbeit geht scheinbar nicht von einer Auseinandersetzung mit diesen Erfahrungen (vgl. hierzu auch: Mecheril, 2008), denn Frau Akay schätzt die Bildungschancen bzw. die Bildungserfolgsaussichten ihrer Schüler*innen insgesamt als eher gering ein. Vor dem Hintergrund der eigenen Aufstiegsbiografie erscheint diese Sichtweise nicht erwartungsgemäß, sie kann aber anhand der institutionalisierten Deutungsmuster, die größtenteils mit negativen stereotypen Vorstellungen von Schüler*innen aus sozial benachteiligten Milieus verbunden sind, erklärt werden. 
Damit wird zu der Notwendigkeit übergeleitet, die problemzentrierten Schüler*innen- und Elternbilder zu reflektieren. Etwa haben die vorgestellten empirischen Studien mit praxeologischem Ansatz deutlich gemacht, dass Normalitätsvorstellungen über angemessene Orientierungen und Praktiken der Schüler*innen und Eltern (vgl. Khan, Sertl, Raggl, Stefan, \& Unterköffler-Klatzer, 2012) sowie stigmatisierende Zuschreibungen entlang sozialer Differenzkategorien (vgl. Fölker \& Hertel, 2015; Jäger, 2014; Wiezorek \& Pardo-Puhlmann, 2013) ausschlaggebende Faktoren für die strukturelle Reproduktion von Bildungsungleichheit darstellen. Lehrkräfte stehen demnach vor der Anforderung, zu hinterfragen, welche Differenzkategorien in welcher Weise innerhalb ihrer pädagogischen Arbeit ungleichheitsrelevant werden. Zentral ist hierbei im Sinne der Sozioanalyse, sich von den vermeintlichen Selbstverständlichkeiten, den unhinterfragt gültigen Wissensbeständen und Begrifflichkeiten im Alltag und im schulischen Feld zu distanzieren (vgl. Brake, 2015). Ansatzweise ist solch ein Bruch mit Common Sense-Theorien bei Frau Kamper und Herrn Jansen rekonstruierbar, die sich zum Teil von pauschalisierenden Defizitzuschreibungen an die Schüler*innen abgrenzen und eine differenzierte Sichtweise auf bestimmte Auffälligkeiten entfalten. Dies zeigt sich an den oben bereits genannten Bezugnahmen auf die vermeintlich verwahrloste Schülerin (Frau Kamper) und den Schüler mit ADHS (Herr Jansen).

Nicht zuletzt muss jedoch festgehalten werden, dass allein die lehrkraftseitigen Bemühungen, sich von schulischen Normen und Konventionen und damit verbundenen Gewohnheiten und Routinen zu befreien, nicht genügen. Um alternative Strukturen und Praktiken des Abbaus von Bildungsungleichheit zu entwickeln, muss darüber hinaus die Schule als Institution selbstkritischer mit der Leistungsund Begabungsideologie und Mechanismen der institutionellen Diskriminierung umgehen (vgl. Solga, 2005; Gomolla \& Radtke, 2009).

\subsection{Grenzen der Untersuchung und anschließende Forschungsperspektiven}

Gleichwohl die vorliegende Studie die bisherige Forschung zu Passungskonstellationen zwischen Lehrkräften und Schüler*innen ungleicher sozialer Herkunft weiter ausdifferenzieren kann, haben sich im Analyseprozess zahlreiche neue Fragen und Themenbereiche aufgetan, die Anlass für weitere Forschungsprojekte geben. Etwa lassen sich anhand der herausgearbeiteten Befunde zwei Grundmuster der lehrkraftseitigen Unterstützung sozial benachteiligter Schüler*innen festmachen, die es tiefer zu ergründen gilt: ein anerzogenes, prinzipiengeleitetes Engagement 
sowie ein aus scheinbar homologen biografischen Erfahrungen resultierendes Engagement. Anschließende Forschungsanstrengungen sollten die Konstruktion konjunktiver Erfahrungsräume, die bislang ausschließlich in den Studien zu beruflichen Selbst- und Fremdkonzepten von Lehrkräften mit Migrationshintergrund untersucht wurden (vgl. Fabel-Lamla \& Klomfaß, 2014; Rotter, 2014; Georgi, Ackermann, \& Karakas, 2011), einbeziehen und die handlungsleitenden milieuspezifischen Orientierungen von Lehrkräften mit und ohne Migrationshintergrund systematisch miteinander vergleichen. Es sollte in breiter angelegten qualitativen Untersuchungen ergänzend bedacht werden, dass sich unterschiedliche milieuspezifische Erfahrungen überlagern, sodass verschiedene Erfahrungsdimensionen zusammenkommen (vgl. hierzu auch: Nohl, 2010).

In dieser Arbeit wurde der Blick auf die Milieuspezifität gerichtet. Darüber hinaus wäre aufschlussreich, die soziogenetische Interpretation auf weitere Erfahrungsräume auszuweiten. Beispielsweise thematisiert Herr Jansen das ungleiche Geschlechterverhältnis der Grundschullehrkräfte und misst sich selbst aufgrund seines männlichen Geschlechts einen Vorteil für die pädagogische Arbeit bei:

also ich sag immer mein-der Job ist für mich ein bisschen einfacher weil gerade die Jungen (.) das hört sich doof an aber ich glaube das ist wirklich so auch ein bisschen deswegen sagt man ja immer mehr Männer in die Grundschulen dass ich den Laden eher ruhig kriege (.) alleine nur weil ich ein Mann bin (Interview Herr Jansen, Z. 2361-2367)

Interessante Anknüpfungspunkte für entsprechende Folgeprojekte bietet mitunter die Studie zum beruflichen Habitus männlicher Grundschullehrer von Baar (2010), in der der Zusammenhang von Handlungsorientierungen und Männlichkeitskonstruktionen herausgearbeitet wird. Ferner können insbesondere bei Frau Berger Geschlechterstereotype (vgl. 2.5.2 Modifikation der Analysedimensionen) identifiziert werden. So beschreibt Frau Berger im Zusammenhang der Frage nach besonderer Sympathie für bestimmte Schüler*innen das positivere Sozialverhalten der Mädchen im Gegensatz zu den Jungen:

oft sind es ja die Mädchen auch nicht immer um Gottes Willen aber oft sind es ja die Mädchen die dann auch so ähm vom Sozialen her so ein bisschen Schlichten und ähm nochmal so alles was hochkocht wieder so ein bisschen runter (.) bringen auch auf-in Schulhofsituationen und und und (.) und (.) ja die einfach im Sozialen auch besonders stark sind da gibt es auch Jungs also es sind jetzt nicht nur Mädchen aber ähm wenn ich jetzt an meine jetzige Klasse denke sind es viele Mädchen (.) und einige Jungs (Interview Frau Berger, Z. 706-715) 
$\mathrm{Zu}$ geschlechtsstereotypen Vorstellungen sowie damit korrespondierenden pädagogischen und unterrichtlichen Praktiken liegen zahlreiche Untersuchungen vor (u. a. Budde, Scholand, \& Faulstich-Wieland, 2008; Budde, 2006; Spitz, 2002). Inwiefern die geschlechtsbezogenen Orientierungen mit milieugeprägten Erfahrungen der Lehrkräfte zusammenhängen, ist hingegen bislang nicht erforscht.

Bei der Eingrenzung der dargestellten Fälle lag der Fokus des Weiteren auf berufseinsteigende Lehrkräfte. Lohnenswert wäre auch eine Gegenüberstellung habitueller Dispositionen von Lehrkräften in unterschiedlichen beruflichen Phasen, um die Wahrnehmung, Einschätzung und Bewältigung von Bildungsungleichheit weiter zu erforschen. Die vorliegende Arbeit kann lediglich über die Dispositionen Auskunft geben, die zum Zeitpunkt des Interviews kommunikativ hervorgebracht wurden. Ein längsschnittlicher Zugang eröffnet ferner die Möglichkeit, Dynamiken der Veränderungen von handlungsleitenden Orientierungen über einen längeren Zeitraum zu analysieren. Anschlussfähig sind hierbei Studien aus berufsbiografischer Perspektive, die die Bewältigung von Entwicklungsaufgaben, Anforderungen und Herausforderungen im Lehrberuf betrachten (u. a. Lamy, 2014; Košinár, 2014; Hericks, 2006). Etwa weist Maschke (2013) in einer Untersuchung von Bildungsverläufen angehender Lehrkräfte darauf hin, dass gerade unter Druck habituelle Wandlungsprozesse stattfinden können. Dabei unterscheidet Maschke defensive und offensive Bewältigungsstrategien von spannungsreichen Situationen, die auf familiale Erfahrungen, soziales Kapital und das Selbstkonzept zurückzuführen sind (ebd.). Erste Anzeichen für derartige (habituelle) Veränderungen von Deutungen und Handlungspraktiken finden sich zum Beispiel in den Interviews mit Frau Kamper und Herrn Jansen, wenn diese ihre individuelle Wahrnehmung und ihren Umgang mit angeblichen Problemschüler*innen im Verhältnis zu ihren Kolleg*innen und in Auseinandersetzung mit den jeweiligen Gegebenheiten vor Ort beschreiben. Demzufolge sollte außerdem ein besonderes Augenmerk auf der Einzelschule selbst liegen. Von Interesse ist vor allem die Identifikation von internen und externen Faktoren, die zu einem Wandel habitueller Dispositionen führen (können). Dazu zählt die Analyse einzelschulischer Umgangsweisen mit sozialer Bildungsungleichheit sowie interaktiver Aushandlungsprozesse hinsichtlich der Positionierung von Lehrkräften ungleicher sozialer Herkunft im Kollegium. In diesem Kontext sollte auch der In- und Außerwertsetzung habitueller Orientierungen von Lehrkräften mehr Aufmerksamkeit gewidmet werden. Untersuchungsleitend könnte für solche zukünftigen Studien die Frage sein, ob Lehrkräfte aus einem eher sozial benachteiligten Herkunftsmilieu, die sich dem Common Sense über institutionell geltende Vorstellungen, 
Regeln und Strukturen widersetzen, eher Gefahr laufen, an den Rand oder sogar aus dem schulischen Feld gedrängt zu werden.

Aussichtsreich erscheint ebenfalls ein Blick über den einzelschulischen Kontext hinaus auf weitere bildungsrelevante Akteure. So kann die Erforschung kommunaler Bildungslandschaften Auskunft darüber geben, inwiefern die Einzelschule in ,langfristige, professionell gestaltete, auf gemeinsames, planvolles Handeln abzielende, kommunalpolitisch gewollte Netzwerke zum Thema Bildung“ (Bleckmann \& Durdel, 2009, S. 12) eingebunden ist. Die systematische Vernetzung und Abstimmung lokaler Bildungsakteure zielt darauf ab, die soziale Selektivität des Bildungssystems abzubauen. Die Frage, auf welche Weise dies in der Praxis gelingen kann, muss jedoch noch näher untersucht werden (vgl. hierzu auch: Duveneck, 2016).

Ferner ist anzumerken, dass durch die rekonstruktive Forschung ein Blick auf die Praxis versperrt bleibt. Mit dieser Arbeit kann nicht beantwortet werden, wie sich die negativen Stereotypisierungen tatsächlich auf die pädagogische Arbeit, bspw. hinsichtlich der Schullaufbahnempfehlungen, auswirken. So kommen über die Interviews ausschließlich die Selbstinterpretationen und Deutungen der befragten Lehrkräfte in den Blick, nicht aber Interaktionen und Praktiken. Dementsprechend wäre eine Kombination von Interviews mit ethnografischen Zugängen wie etwa teilnehmende Beobachtungen aufschlussreich, um zusätzlich die Wirksamkeit und Wirkungsrichtung der handlungsleitenden Orientierungen abzubilden und schulische Interaktionsprozesse zwischen verschiedenen Akteuren innerhalb und außerhalb des Unterrichts in den Blick zu nehmen.

Vielversprechend erscheint überdies die Anbindung an die pädagogische Professionsforschung. Aus den in dieser Arbeit generierten Befunden lassen sich insbesondere zwei Ansatzpunkte für weitere Untersuchungen ableiten:

(1) Erstens sollte die Genese (und wie oben bereits genannt: mögliche Veränderungen) habitueller Orientierungen von Lehrkräften als noch unerschlossenes Forschungsgebiet fokussiert werden. Unterdies verweisen Bressler und Rotter (2018) auf die doppelte theoretische Bedeutung des Habituskonzepts und plädieren für die Erweiterung der Forschung zum Habitus von Lehrkräften - nicht nur im Hinblick auf eine ganzheitliche Theoriebildung, sondern auch in Bezug auf die praktische Relevanz für die Lehrer*innenbildung. Den Autor*innen zufolge kann ein tiefergehendes Verständnis des Habitus als ,Modus Operandi ‘ Auskunft über Bedarfe der Professionalisierung des beruflichen Habitus geben; eine Beforschung des Habitus als ,Opus Operatum “ lässt hingegen Rückschlüsse über Grenzen und Möglichkeiten der Professionalisierung des beruflichen Habitus zu (ebd.). Mit diesen Überlegungen 
zusammenhängend steht auch der Modellentwurf der Herausbildung habitueller Orientierungen von Lehrkräften. So schlägt Helsper (2018a; 2018b) die Unterscheidung zwischen (1) dem familiären, primären Habitus, (2) dem biografisch erworbenen, individuellen Habitus, (3) dem Schüler*innenhabitus und (4) dem Lehrer*innenhabitus vor. Helsper argumentiert, dass die impliziten Orientierungen und Praktiken, die während der eigenen Schulzeit erworben wurden, - auch in Korrespondenz zu dem familiären, primären Habitus und dem biografisch erworbenen, individuellen Habitus - in die Entwicklung eines eigenen Lehrer*innenhabitus eingehen.

(2) $\mathrm{Zu}$ der Ausdifferenzierung des Habitusbegriffs tritt zweitens die begrifflichkonzeptionelle Schärfung des Verhältnisses von Lehrer*innenhabitus und Professionalität hinzu. So gibt Helsper zu bedenken, dass mit dem Begriff des Lehrer*innenhabitus in einer ausschließlich empirischen Perspektive danach gefragt wird, welche unterschiedlichen Dispositionen rekonstruierbar sind. Mit dem Begriff der Professionalität wird hingegen ein normativer Horizont eröffnet, sodass die habituell verankerten Orientierungen und Praktiken daraufhin befragt werden können, inwiefern sie den Anforderungen an eine professionelle Handlungspraxis entsprechen (ebd.). Kramer und Pallesen (2018) entwickeln hierzu auf Grundlage des strukturtheoretischen Professionsansatzes (u. a. Helsper, 2011; Oevermann, 2002, 1996) eine Heuristik des professionellen Lehrer*innenhabitus. Unter der zentralen Handlungsanforderung, Anregung, Wahrnehmung und Begleitung von Bildungsprozessen über paradoxe Kriseninterventionen' zeichnet sich ein professioneller Habitus der Lehrkräfte vor allem über die Berücksichtigung der Widersprüchlichkeiten der beabsichtigten Autonomieförderung und deren gleichzeitiger heteronomen Beeinträchtigung durch das eigene Lehrer*innenhandeln aus. $\mathrm{Zu}$ der Handlungsanforderung ,Gestaltung eines pädagogischen Arbeitsbündnisses “ zählt im Sinne von Professionalität die situative Verortung der verschiedenen universalen und partikularen Facetten eines pädagogischen Arbeitsbündnisses im eigenen Handeln. Unabdingbare Anforderung für den Lehrberuf ist darüber hinaus die Fähigkeit zum Fallverstehen. Professionell wäre ein Lehrer*innenhabitus dann, wenn eine situative Einbettung des Einzelfalls in theoretische Kategorien bei gleichzeitiger Vermeidung von schematischen Abläufen gelingt. Alle bisher genannten Anforderungsbereiche setzen eine grundlegende Reflexionsbereitschaft und -fähigkeit voraus. Professionalität bedeutet in diesem Zusammenhang einen reflexiven Umgang mit Ungewissheit sowie der Unsicherheit der eigenen Wissensbasis und deren Relevanz für konkrete Fälle (ebd.). 
Zum Schluss dieser Arbeit bleibt festzuhalten, dass die in den vorherigen Kapiteln berichteten Ergebnisse und die in diesem Kapitel skizzierten Handlungsoptionen, weiterführende Fragen und Themenbereiche wesentlich zu einer selbstkritischen Reflexion der pädagogischen Arbeit und somit zum produktiveren Umgang mit herkunftsbedingter Vielfalt beitragen können. Das Erkennen der ,,verborgenen Mechanismen der Macht“ (Bourdieu, 1992) im schulischen Feld erlaubt es den Lehrkräften, die eigenen Potenziale zu stärken und Handlungsmöglichkeiten zur Unterstützung sozial benachteiligter Schüler*innen zu entwickeln.

Open Access Dieses Kapitel wird unter der Creative Commons Namensnennung 4.0 International Lizenz (http://creativecommons.org/licenses/by/4.0/deed.de) veröffentlicht, welche die Nutzung, Vervielfältigung, Bearbeitung, Verbreitung und Wiedergabe in jeglichem Medium und Format erlaubt, sofern Sie den/die ursprünglichen Autor(en) und die Quelle ordnungsgemäß nennen, einen Link zur Creative Commons Lizenz beifügen und angeben, ob Änderungen vorgenommen wurden.

Die in diesem Kapitel enthaltenen Bilder und sonstiges Drittmaterial unterliegen ebenfalls der genannten Creative Commons Lizenz, sofern sich aus der Abbildungslegende nichts anderes ergibt. Sofern das betreffende Material nicht unter der genannten Creative Commons Lizenz steht und die betreffende Handlung nicht nach gesetzlichen Vorschriften erlaubt ist, ist für die oben aufgeführten Weiterverwendungen des Materials die Einwilligung des jeweiligen Rechteinhabers einzuholen.

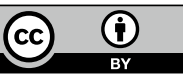

